\title{
Status of the CEPC Project: Physics, Accelerator and Dectector
}

\author{
Jie Gao*i \\ Institute of High Energy Physics, Beijing, China \\ E-mail: gaojeihep.ac.cn
}

\begin{abstract}
In this paper we will give an introduction to Circular Electron Positron Collider (CEPC). The scientific background, physics goal, the collider design requirements and the conceptual design principle of CEPC are described. On CEPC accelerator, the optimization of parameter designs for CEPC with different energies, machine lengthes, single ring and crab-waist collision partial double ring options, etc. have been discussed systematically. The sub-systems of CEPC, such as collider main ring, booster, electron positron injector, etc. have been introduced. The detector and MDI design have been briefly mentioned. Finally, the optimization design of Super ProtonProton Collider (SPPC), its energy and luminosity potentials, in the same tunnel of CEPC are also discussed.
\end{abstract}

38th International Conference on High Energy Physics

3-10 August 2016

Chicago, USA

\footnotetext{
* Speaker.

$\dagger$ This study was supported by National Key Programme for S\&T Research and Development (Grant NO.: 2016YFA0400400) and National Natural Science Foundation of China (NO.11575218, 11505198, 11605211).
} 


\section{Introduction}

With the discovery of the Higgs particle at the Large Hadron Collider at CERN in July 2012, after more than 50 years of searching, particle physics has finally entered the era of the Higgs, and the door for human beings to understand the unknown part of the Universe is wide open! Thanks to the low energy of Higgs, it is possible to produce clean Higgs with circular electron positron colliders in addition of linear colliders, such as ILC and CLIC, with reasonable luminosity, technology, cost, and power consumption.

In September 2012, Chinese scientists proposed a Circular Electron Positron Collider (CEPC) in China at $240 \mathrm{GeV}$ centre of mass for Higgs studies with two detectors situated in a very long tunnel more than twice the size of the LHC at CERN. It could later be used to host a Super Proton Proton Collider (SppC) well beyond LHC energy potential to reach a new energy frontier in the same channel.

After ICFA Higgs Factory Workshop held at Fermi Laboratory in Nov 2012, CERN proposed also a similar one, Future Circular Collider (FCC) with a much longer tunnel than that of LHC. From 12 to 14 June 2013, the 464th Fragrant Hill Meeting was held in Beijing on the strategy of Chinese high energy physics development after Higgs discovery, and the following consensuses were reached: 1) support ILC and participate to ILC construction with in kind contributions, and request R\&D fund from Chinese government; 2) as the next collider after BEPCII in China, a circular electron positron Higgs factory (CEPC) and a Super proton-proton Collier (SppC) afterwards in the same tunnel is an important option as a historical opportunity, and corresponding R\&D is needed. ICFA has given two successive statements in Feb. and July of 2014, respectively, that ICFA supports studies of energy frontier circular colliders and encourages global coordination; ICFA continues to encourage international studies of circular colliders, with an ultimate goal of proton-proton collisions at energies much higher than those of the LHC. During the AsiaHEP and ACFA meeting in Kyoto in April 2016, a positive statement of AsiaHEP/ACFA Statement on ILC+CEPC/SppC has been made with strong endorsement of the ILC and encouraging the effort led by China on CEPC/SppC. On Sept 12, 2016, during the meeting of the Chinese High Energy Physics of Chinese Physics Society, a statement on the future Chinese high energy physics based on accelerator has been made that CEPC is the first option for future high energy accelerator project in China as a strategic action with the aim of making CEPC as a large international scientific project proposed by China. The 572th Fragrant Hill Meeting dedicated to CEPC has been held from Oct. 18-19, 2016, and it is concluded that CEPC has a solid physics reason to be built with big physics potential in SppC. The optimization design, relevant technologies and industry preparation could be ready after a five years dedicated R\&D period before CEPC starts to be constructed around 2022 and completed around 2030. CEPC will operate 10 ten years with two detectors to accumulate one million Higgs and 100 million of $\mathrm{Z}$ particle.

In the beginning of 2015, Pre-Conceptual Design Reports (Pre-CDR) of CEPC-SppC [1] have been completed with international review. The International Advisory Committee (IAC) of CEPC was also established in 2015. At the end of 2016 a CDR Status Report will be finished before finishing of the CDR at the end of 2017. In 2016, Chinese Ministry of Science and Technology has allocated several tens of million RMB on CEPC R\&D to start with. 


\section{CEPC accelerator design}

According to the physics goal of CEPC at Higgs and Z-pole energy, it is required that the CEPC provides $\mathrm{e}^{+} \mathrm{e}^{-}$collisions at the center-of-mass energy of $240 \mathrm{GeV}$ and delivers a peak luminosity of $2 \times 10^{34} \mathrm{~cm}^{-2} \mathrm{~s}^{-1}$ at each interaction point. CEPC has two IPs for $\mathrm{e}^{+} \mathrm{e}^{-}$collisions. At Z-pole energy the luminosity is required to be larger than $1 \times 10^{34} \mathrm{~cm}^{-2} s^{-1}$ per IP. Its circumference is around 60 $\mathrm{km}$ in accordance with $\mathrm{SppC}$, which has $70 \mathrm{TeV}$ of center of mass proton proton collision and 20 Tesla superconduction magnet dipole field. The schematic layout of CEPC-SppC is shown in Fig. 1, and CEPC accelerator complex is composed of a $6 \mathrm{GeV}$ electron and positron linac injector with a $1 \mathrm{GeV}$ psotron damping ring, a booster from $6 \mathrm{GeV}$ to $120 \mathrm{GeV}$ in the same channel of $120 \mathrm{GeV}$ collider rings.

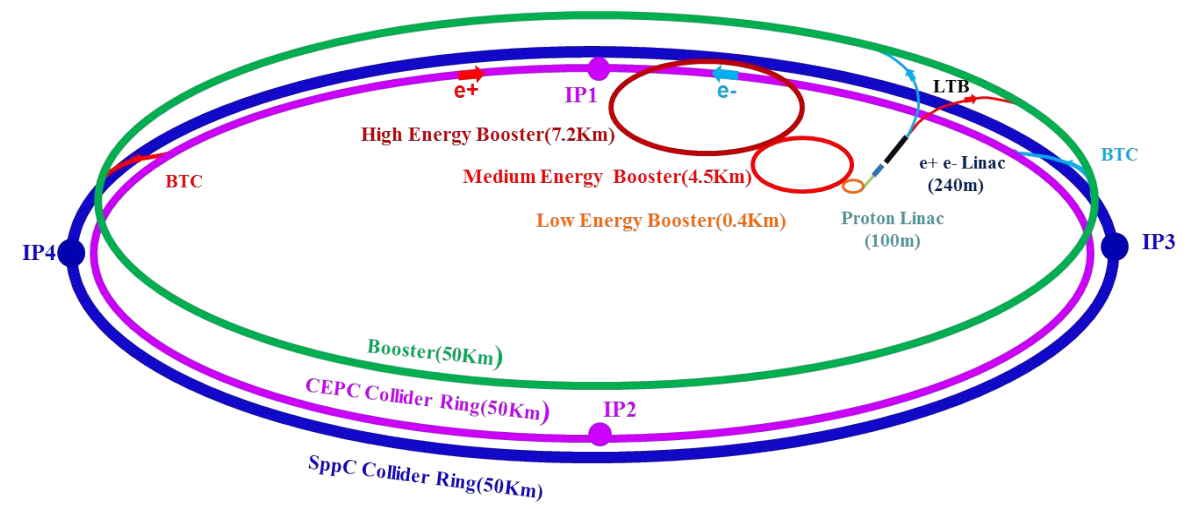

Figure 1: CEPC-SPPC schematic layout.

\subsection{Main parameters and main ring designs}

To make an optimization a collider, started from the goals, such as energy, luminosity/IP, number of IPs, etc, one has to consider very key beam physics limitations, such as beam-beam effects [2] and Beamstrahlung [3], and also take into account of economical and technical limitations, such synchrotron radiation power and high order mode power in each Superconducting rf cavity. By taking into account all these limitations in an analytical way, an analytical electron positron circular collider optimized design methods have been developed both head-on collision and ccarb-waist collision. The CEPC parameters of single ring head-on collision scheme as used in CEPC-SppC Pre-CDR and the crab-waist collision designs are shown in Tab. 1 [4].

In Pre-CDR, single ring head-on collision scheme has been studied with Pretzel scheme. The apparent low cost single ring Pretzel scheme has many problems, such as not flexible lattice solution, small dynamic aperture, low Z-pole energy luminosity (around $10^{32} \mathrm{~cm}^{-2} \mathrm{~s}^{-1}$ ), and very high AC power consumption (around 500MW). To solve these critical problems, a Partial Double Ring (PDR) scheme has been proposed independently [5][6]. In Tab. 1 we could find that with crab wait collision, one could reduce synchrotron radiation power from $50 \mathrm{MW}$ to about $30 \mathrm{MW}$, and with Z-pole luminosity to satisfy the design requirement. In fact, in addition to single ring and partial double ring schemes, there are two other types of schemes, i.e. Advanced Partial Double 
Table 1: Main parameters of CEPC

\begin{tabular}{|c|c|c|c|c|c|}
\hline & Pre-CDR & H-high Iumi. & H-low power & $w$ & $Z$ \\
\hline Number of IPs & 2 & 2 & 2 & 2 & 2 \\
\hline Energy $(\mathrm{GeV})$ & 120 & 120 & 120 & 80 & 45.5 \\
\hline Circumference $(\mathrm{km})$ & 54 & 61 & 61 & 61 & 61 \\
\hline SR loss/turn $(\mathrm{GeV})$ & 3.1 & 2.96 & 2.96 & 0.58 & 0.061 \\
\hline Half crossing angle (mrad) & 0 & 15 & 15 & 15 & 15 \\
\hline Piwinski angle & 0 & 1.88 & 1.84 & 5.2 & 6.4 \\
\hline$N_{e}$ /bunch $\left(10^{11}\right)$ & 3.79 & 2.0 & 1.98 & 1.16 & 0.78 \\
\hline Bunch number & 50 & 107 & 70 & 400 & 1100 \\
\hline Beam current $(\mathrm{mA})$ & 16.6 & 16.9 & 11.0 & 36.5 & 67.6 \\
\hline SR power /beam (MW) & 51.7 & 50 & 32.5 & 21.3 & 4.1 \\
\hline Bending radius $(\mathrm{km})$ & 6.1 & 6.2 & 6.2 & 6.2 & 6.2 \\
\hline Momentum compaction $\left(10^{-5}\right)$ & 3.4 & 1.48 & 1.48 & 1.44 & 2.9 \\
\hline$\beta_{I P} \mathrm{x} / \mathrm{y}(\mathrm{m})$ & $0.8 / 0.0012$ & $0.272 / 0.0013$ & $0.275 / 0.0013$ & $0.1 / 0.001$ & $0.1 / 0.001$ \\
\hline Emittance $\mathrm{x} / \mathrm{y}(\mathrm{nm})$ & $6.12 / 0.018$ & $2.05 / 0.0062$ & $2.05 / 0.0062$ & $0.93 / 0.0078$ & $0.88 / 0.008$ \\
\hline Transverse $\sigma_{I P}(\mathrm{um})$ & $69.97 / 0.15$ & $23.7 / 0.09$ & $23.7 / 0.09$ & $9.7 / 0.088$ & $9.4 / 0.089$ \\
\hline$\xi_{x} / \mathrm{IP}$ & 0.118 & 0.041 & 0.042 & 0.013 & 0.01 \\
\hline$\xi_{y} / \mathrm{IP}$ & 0.083 & 0.11 & 0.11 & 0.073 & 0.072 \\
\hline$V_{R F}(\mathrm{GV})$ & 6.87 & 3.48 & 3.51 & 0.74 & 0.11 \\
\hline$f_{R F}(\mathrm{MHz})$ & 650 & 650 & 650 & 650 & 650 \\
\hline Nature $\sigma_{z}(\mathrm{~mm})$ & 2.14 & 2.7 & 2.7 & 2.95 & 3.78 \\
\hline Total $\sigma_{z}(\mathrm{~mm})$ & 2.65 & 2.95 & 2.9 & 3.35 & 4.0 \\
\hline HOM power/cavity (kw) & 3.6 & 0.74 & 0.48 & 0.88 & 0.99 \\
\hline Energy spread (\%) & 0.13 & 0.13 & 0.13 & 0.087 & 0.05 \\
\hline Energy acceptance (\%) & 2 & 2 & 2 & & \\
\hline Energy acceptance by RF (\%) & 6 & 2.3 & 2.4 & 1.7 & 1.2 \\
\hline$n_{\gamma}$ & 0.23 & 0.35 & 0.34 & 0.49 & 0.34 \\
\hline $\begin{array}{l}\text { Life time due to } \\
\text { beamstrahlung_cal (minute) }\end{array}$ & 47 & 37 & 37 & & \\
\hline$F$ (hour glass) & 0.68 & 0.82 & 0.82 & 0.92 & 0.93 \\
\hline$L_{\max } / \mathrm{IP}\left(10^{34} \mathrm{~cm}^{-2} \mathrm{~s}^{-1}\right)$ & 2.04 & 3.1 & 2.01 & 4.3 & 4.48 \\
\hline
\end{tabular}

Ring (APDR) [7] and Double Ring (DR) scheme [8]. In fact, in principle, the crab-waist CEPC parameters could be realized by PDR, APDR and DR schemes. PDR, APDR and DR are also called options to a crab-waist collision scheme. However, if one take synchrotron radiation effect and the collective effect of superconducting accelerator system taking into account, the three options are quite different from one from another. Apparently, DR is the most expensive and relative easy option, APDR as shown in Fig. 2(PDR is a special case of APDR, only two partial double ring sections at two IPs) is most possible economic option overcoming the difficulties from PDR, i.e., beam loading and sawtooth effects, which should be studied carefully before a reasonable choice among differen options.

As for PDR (APDR) lattice design, in the Arc region, the FODO cell structure is chosen to provide a large filling factor. The $90 / 90$ degrees phase advances is chosen to achieve a very small emittance of $2 \mathrm{~nm}$. The non-interleaved sextupole scheme [9] was selected due to its property of small tune shift. Considering the symmetry of two IPs and two beams, the lattice CEPC PDR scheme has a four-fold symmetry and the maximum number of sextupole families in the ARC region is 96 [10].

The CEPC interaction region (IR) was designed with modular sections including the final transformer, chromaticity correction for vertical plane, chromaticity correction for horizontal plane and matching transformer. To achieve a momentum acceptance as large as $2 \%$, local correction of the large chromaticity from final doublet is necessary.

The dynamic aperture of the ring is optimized by SAD and goal is to have dynamic aperture in both transverse planes lager than $5 \sigma$ including all effects with energy spread of from $+2 \%$ to 


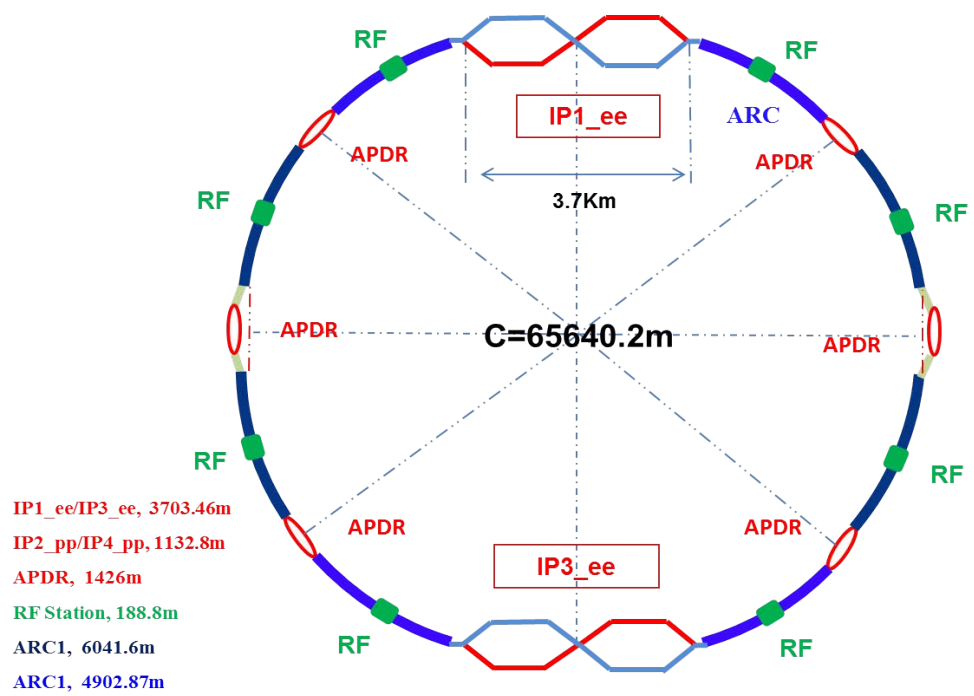

Figure 2: CEPC advanced partial double ring scheme.

$-2 \%$.

\subsection{Injector}

To reduce the cost of the whole system, the length of the Linac is chosen to be as short as possible, and a booster ring is used to ramp the beams from the Linac energy to the full injection energy of the main collider. Therefore, the whole CEPC system is composed of three parts: a linac, a booster, the main collider ring. The Linac injector system is composed of a $6 \mathrm{GeV} \mathrm{S-band} \mathrm{linac}$ with positron source and a $1 \mathrm{GeV}$ damping ring.

\subsubsection{Booster}

The booster provides $120 \mathrm{GeV}$ electron and positron beams to the CEPC collider for top-up injection at $0.1 \mathrm{~Hz}$. The Booster is in the same tunnel as the collider, placed above the collider ring and has about same circumference. The design of the full energy booster ring of the CEPC is especially challenging due to the injected beam only $6 \mathrm{GeV}$, which might cause difficulties. As an alternative design we studied also a wiggler dipole magnets to raise the initial magnetic field [11].

\subsection{Detector and MDI}

The CEPC conceptual detector takes the ILD detector as starting point [12][13]. Similar to the ILD, the core part of this conceptual detector is a solenoid with 3.5 Tesla Magnet Field. To minimize the dead zone, the entire ECAL, HCAL and the tracking system are installed inside the solenoid. The tracking system is composed of a large volume TPC as the main tracker and the silicon tracking system. The interaction region of the CEPC partial double ring consists of two beam pipes, of which the crossing angle is $30 \mathrm{mrad}$, surrounded by silicon tracker, luminosity calorimeter and the final quadrupoles QD0 and QF1, with $\mathrm{L}^{*}$ is $1.5 \mathrm{~m}$ [14]. The inner radius of the

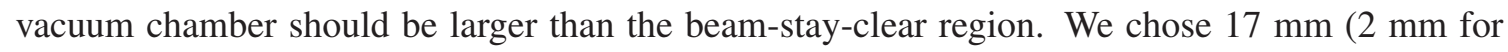
safety) both for QD0 and QF1. 


\section{SPPC design}

The design goal of the SPPC is about $70 \mathrm{TeV}$, using the same tunnel as the CEPC of $61 \mathrm{~km}$, with SC dipole magnet field of about 20 Tesla of luminosity of $1.2 \times 10^{35} / \mathrm{cm}^{-1} \mathrm{~s}^{-1}$. If $100 \mathrm{~km}$ ring is adopted a proton beam of $128 \mathrm{TeV}$ of luminosity of $1 \times 10^{36} / \mathrm{cm}^{-1} \mathrm{~s}^{-1}$ at 20 Tesla could be obtained, and parameter choice and optimization process is given in Ref. [15].

\section{Conclusions}

In this paper we have briefly reviewed the CEPC-SppC projects history, design philosophy and actual status. A dedicated R\&D program both on accelerator and detectors has started with support of Chinese MOST.

The author thanks CEPC-SppC design groups' hard works and their international collaborators's contributions.

\section{References}

[1] The CEPC-SPPC Study Group. "CEPC-SPPC Preliminary Conceptual Design Report, Volume II-Accelerator". IHEP-AC -2015-01, March 2015.

[2] J. Gao, "Review of some important beam physics issues in electron positron collider designs", Modern Physics Letters A, Vol. 30, No. 11, p. 1530006, 2015.

[3] V. Telnov, arXiv:1203.6563v, 29 March 2012.

[4] D. Wang, J. Gao et al.. "CEPC partial double ring scheme and crab-waist parameters". International Journal of Modern Physics A, Vol. 31, (2016) 1644016.

[5] J. Gao, IHEP-AC-LC-Note 2013-012.

[6] M. Moratzinos and F. Zimmermann, "Mitigatng performance limitations of single beam pipe circuar e+e- colliders", IPAC 2015.

[7] J. Gao, "The advanced partial double ring scheme for CEPC",IHEP-AC-LC-Note2016-002.

[8] K. Oide, "FCC(ee) Status", in this Proceedings.

[9] Yukiyoshi Ohnishi et. al. "Accelerator design at SuperKEKB". Prog. Theor. Exp. Phys. 2013, 03 A011.

[10] Yiwei Wang et.al. "Dynamic Aperture study of the CEPC main Ring with Interaction Region". THPOR012, IPAC2016.

[11] T.J. Bian, J. Gao, et al, "Design study of CEPC alternation magnetic field booster", THPOR011, IPAC2016.

[12] The CEPC-SPPC Study Group. "CEPC-SPPC Preliminary Conceptual Design Report, Volume I-Physics and Detector". IHEP-EP -2015-01, March 2015.

[13] M. Ruan, "Higgs measurements at e+e- Circular Colliders", Proceeding of ICHEP 2014, arXiv $1411.5606 \mathrm{v} 1$.

[14] Sha Bai et.al. "MDI Design in CEPC partial double ring". THPOR014, IPAC2016.

[15] F. Su, J. Gao et al, "SPPC Parameter Choice and Lattice Design", TUPMW001, Proceedings of IPAC2016. 